\title{
UK Renal Registry 15th Annual Report: Chapter 8 UK Multisite Peritoneal Dialysis Access Catheter Audit for First PD Catheters 2011
}

\author{
Victoria Briggs ${ }^{a}$, David Pitcher ${ }^{b}$, Fiona Braddon ${ }^{b}$, Damian Fogarty $^{b, c}$, Martin Wilkie $^{a}$

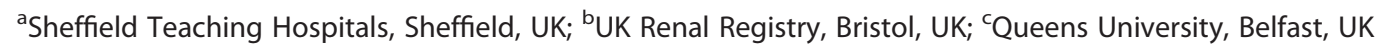

\section{Key Words}

Access - Catheter outcome - Deprivation - Peritoneal dialysis

- Primary renal disease

\begin{abstract}
08
Background: The central paradigm of effective peritoneal dialysis (PD) is an appropriate standard of PD catheter function. Aim: The aim of the project was to develop an effective national PD access audit which would identify an 'appropriate standard' of PD catheter function. Methods: The UK Renal Registry collected centre specific information on various PD access outcome measures including catheter functionality and post-insertion complications. The first PD access audit covering England, Northern Ireland and Wales was conducted during April to June 2012 looking at incident dialysis patients in 2011. Results: Forty three
\end{abstract}

data collection spreadsheets were returned from a total of 65 centres describing 917PD catheter placements. The median age of PD patients was 61 years and 61.5\% were male. The proportion of patients initiated on PD in comparison to HD was lower in socially deprived areas. There was a relationship between the timing of nephrology referral and the likelihood of surgical assessment regarding PD catheter placement. Patients with diabetes did not have higher rates of PD catheter failure or of early peritonitis. Conclusions: A comparative PD catheter audit has the potential to provide valuable information on an important patient related outcome measure and lead to an improvement in patient experience. There was wide variation between centres of PD catheter use for late presenting patients. Overall patients were more likely to get a PD catheter if they had been known to the service for more than 1 year. The percutaneous insertion technique was associated with a higher early (less than 2 week) peritonitis rate and more catheter flow problems.

\section{KARGER}

Fax +4161306 1234 E-Mail karger@karger.ch www.karger.com
Victoria Briggs

UK Renal Registry, Southmead Hospital, Southmead Road, Bristol, BS10 5NB, UK

Email: renalregistry@renalregistry.nhs.com 


\section{Introduction}

Peritoneal dialysis (PD) is a key mode of renal replacement therapy (RRT) being used by $18 \%$ of United Kingdom (UK) dialysis patients [1]. In order for PD to be both clinically successful and acceptable from the perspective of patient experience, sustained catheter function in the absence of significant complications is essential. Poorly functioning PD catheters prevent patients from getting the best from renal replacement therapy and poor catheter function often leads to the abandonment of the modality completely. Surprisingly, such information has not been routinely collected from UK renal centres until now.

Whilst published guidelines relating to PD catheter functionality and post-insertion complication thresholds exist, (International Society for Peritoneal Dialysis (ISPD) [2] and the Renal Association (RA) [3]) their validity has not been rigorously evaluated. The accurate collection of PD access data remains a challenge due to ambiguity of data terms and the methodology of data collection. This is exacerbated by the presence of untested audit standards [4]. Two important audit standards which have emerged from current RA/ISPD guidance are:

1) Peritonitis rate occurring within 2 weeks of catheter insertion should occur in less than $5 \%$ of cases

2) Catheter patency should be more than $80 \%$ at 1 year. This report does not capture this length of follow up.

The requirement for timely peritoneal dialysis access is also of paramount importance and is described in the Renal Association Peritoneal Dialysis clinical guideline 2.1[5]:

\section{'Fast track education and urgent PD catheter inser- tion with acute start PD should be available, and be offered to suitable patients urgently starting on renal replacement therapy who wish to avoid tempor- ary haemodialysis'.}

The associated audit measures describe the care pathway for catheter insertion including timeliness and requirement for temporary haemodialysis. Further audit measures describe catheter complications and their resolution. In order to advance this important area of clinical care, funding was received from the Health Quality Improvement Partnership (HQIP) to enable the UK Renal Registry to initiate a process of data acquisition relating to $\mathrm{PD}$ functionality and access. This report describes selected observations from the first round of data collection from incident PD patients in 2011.

\section{Methodology}

The work supported by HQIP was described in a previous report [6]. All adult renal centres were contacted regarding vascular and peritoneal access in all new patients in 2011. Of 65 centres contacted, data were received from 43 centres. These centres contributed incident peritoneal dialysis access data during 2011 relating to first PD episode (i.e. first PD catheter) in 917 patients ( 353 females, 563 males) (one patient was excluded as under the age of 16 when PD started). Catheter insertion technique was reported as percutaneous in 240 patients, open surgery in 409, laparoscopic in 111, peritoneoscopic in 33 and missing in 124.

Data fields were refined from existing renal registry tables, adjusted based on audit work conducted in Yorkshire and the Humber during 2010 and meetings of the multisite audit group which included patient representation. Data were collected using Excel spreadsheets circulated by the UK Renal Registry. The records collected by the questionnaires were matched with the UK Renal Registry database allowing identification of unreported deaths within three months of commencing dialysis and patients who had previously received RRT.

Referral time was defined as the time between the date of first being seen by a renal physician and the date of commencing dialysis. A valid referral time was calculated for a patient if they had both dates recorded and if the date of first being seen by a renal physician was no later than the date of commencing dialysis. Two centres had no valid referral times calculated for any of their patients due to poor data completeness. If a patient did not have a date that they were first seen by a renal physician available, then the data field should have been left blank. However, patients from London St Bart's \& The London Hospital for whom this data were unavailable had had this date recorded as the date they started dialysis. For this reason, when the data were validated, all 11 patients from London St Bart's \& The London Hospital who had matching dates for these two data fields had the date they were first seen by a renal physician set to missing. This might have caused an under estimation of the number of late referrals at London St Bart's \& The London Hospital as some of the dates that were changed may have been accurate.

Deprivation quintiles were calculated using the English Indices of Deprivation 2010 which measured relative levels of deprivation in small areas of England called Lower Layer Super Output Areas (English Indices of Deprivation 2010: http://www.communities.gov.uk/publications/corporate/statistics/ indices2010). These 32,482 areas were ranked from least deprived to most deprived and then split into equal quintiles. Patient records were matched to an area, and accordingly a deprivation quintile, by postcode. Only patients resident in England with a 
valid postcode were included in the analyses involving deprivation quintiles.

Catheter survival at 3 months was censored for death, transplantation, stopping treatment, and switching to haemodialysis (HD) with no catheter failure. It was not possible to compare centres regarding 3 month catheter function as a measure of the success of catheter placement due to the small number of catheters inserted at each centre.

Patients were classified according to the length of time they were known to nephrology services: less than 90 days, 90 days to 1 year and more than 1 year. This audit reports the commonly used PD catheter insertion methods in the UK as described in the RA PD access working party report [7] and summarised as:

- an open surgical approach in which the layers of the abdominal wall are opened under direct vision and the catheter placed at laparotomy

- a percutaneous Seldinger approach

- placement using a peritoneoscope

- placement aided by a laparoscope

Data completeness by centre ranged from $0 \%$ to $100 \%$ for almost all of the data fields that were collected, including the date the catheter was first used, catheter insertion technique, access at three months, date of catheter failure, BMI and date first seen by renal physician. Statistical analyses were performed using SAS 9.2.

\section{Results}

Table 8.1 shows patient demographic data and percentage completeness of the data items collected.

\section{Demography and primary renal disease}

The majority of peritoneal dialysis patients in the audit were male ( $\mathrm{n}=563,61.5 \%)$ compared to females $(\mathrm{n}=353,38.5 \%)$. This trend was reflected in all age groups except the youngest (figure 8.1). The peak age range for incident patients was 65 to 79 years for both male and female patients, re-enforcing findings in the recent vascular access report [8].

The median age at first dialysis across all United Kingdom centres was 61 years but varied widely across centres. The lowest median age was 33.8 in Tyrone and increased to a maximum median age of 79.7 in Glan Clwyd (figure 8.2).

The most common underlying primary renal disease (PRD) in incident peritoneal dialysis patients (with first PD catheter inserted in 2011) was diabetes mellitus $(21 \%)$, with glomerular disease $(13 \%)$, polycystic kidney disease $(9 \%)$ and hypertension (7\%) representing other

Table 8.1. Demographic data for patients included in the PD access audit

\begin{tabular}{|c|c|c|c|c|}
\hline & $\%$ complete & Data item & $\begin{array}{c}\mathrm{N} \\
916 \\
\text { patients }\end{array}$ & $\begin{array}{c}\% \\
\text { of completed } \\
\text { records }\end{array}$ \\
\hline Gender & 100 & $\begin{array}{l}\text { Male } \\
\text { Female }\end{array}$ & $\begin{array}{l}563 \\
353\end{array}$ & $\begin{array}{l}61.5 \\
38.5\end{array}$ \\
\hline Diabetes at time of catheter insertion & 77 & $\begin{array}{l}\text { Yes } \\
\text { No }\end{array}$ & $\begin{array}{l}224 \\
483\end{array}$ & $\begin{array}{l}31.7 \\
68.3\end{array}$ \\
\hline First modality & 100 & $\begin{array}{l}\text { PD } \\
\text { HD }\end{array}$ & $\begin{array}{r}867 \\
49\end{array}$ & $\begin{array}{r}94.7 \\
5.3\end{array}$ \\
\hline Catheter failure & 14 & Date recorded & 127 & 100.0 \\
\hline BMI & 38 & $\begin{array}{l}\text { Underweight } \\
\text { Normal } \\
\text { Overweight } \\
\text { Obese }\end{array}$ & $\begin{array}{r}10 \\
131 \\
125 \\
79\end{array}$ & $\begin{array}{r}2.9 \\
38.0 \\
36.2 \\
22.9\end{array}$ \\
\hline Primary renal disease & 100 & $\begin{array}{l}\text { Diabetes } \\
\text { Glomerulonephritis } \\
\text { Hypertension } \\
\text { Other } \\
\text { Polycystic kidney } \\
\text { Pyelonephritis } \\
\text { Renal vascular disease } \\
\text { Uncertain aetiology } \\
\text { Missing }\end{array}$ & $\begin{array}{r}194 \\
117 \\
65 \\
88 \\
87 \\
59 \\
34 \\
152 \\
120\end{array}$ & $\begin{array}{r}21.2 \\
12.8 \\
7.1 \\
9.6 \\
9.5 \\
6.4 \\
3.7 \\
16.6 \\
13.1\end{array}$ \\
\hline & & & Mean & SD \\
\hline Age & 100 & At start of dialysis & 58.6 & 16.7 \\
\hline
\end{tabular}




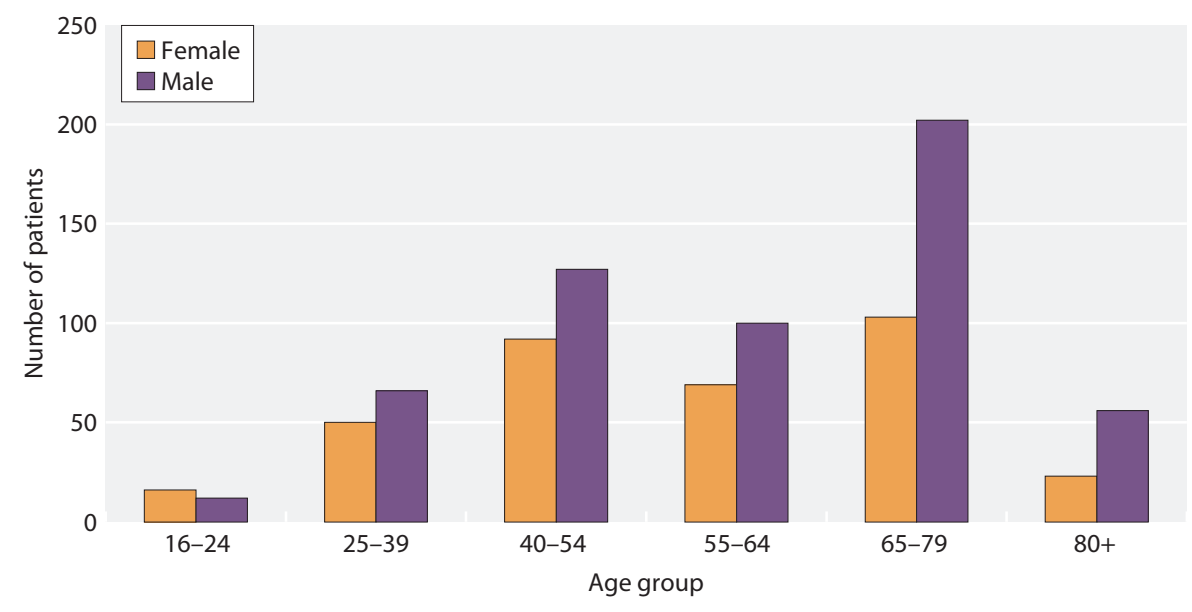

Fig. 8.1. Age and gender of PD patients submitted to audit notable primary renal diseases; $17 \%$ of patients lacked a clear underlying primary renal disease and data were not available for $13 \%$ of patients (figure 8.3, table 8.1).

Diabetes mellitus as the primary aetiological renal disease in PD patients was notably over represented in the most deprived quintile (26.7\%) when compared to the least deprived (13.3\%) quintile. Interestingly, similar trends, either directly or inversely, were not apparent for other primary renal diseases such as hypertension or glomerular diseases (figure 8.4).

Figure 8.5 shows a greater proportion of PD patients in England were derived from the most deprived quintile of the population ( $16 \%$ for the least versus $24 \%$ for the most). This was consistent with a greater incidence of end stage kidney disease in the most deprived groups of the UK population [9].

Although absolute dialysis patient numbers increased with increasing deprivation, PD as a modality fell proportionally across deprivation quintiles with the most deprived patient cohort being the least likely to use PD as a renal replacement modality (25\% for the least deprived versus $19 \%$ for the most deprived quintile) (figure 8.6). Data were stratified by referral time to see if there were confounding factors but for patients who were referred more than 90 days prior to the start of RRT there was still a marked difference between the deprivation quintiles. For late presenters, all quintiles had roughly equal proportions of $\mathrm{HD}$ and $\mathrm{PD}$ patients (figure 8.7).

\section{Impact of referral interval on PD uptake and catheter} placement method

The proportion of PD patients who were late presenters $(<90$ days between presentation and initiation of therapy), varied markedly between renal networks (figure 8.8). Late presenting patients accounted for $15 \%$ of all PD patients in the West Midlands contrasting with other networks such as the South West where only $2 \%$ of PD patients were late presenters. Data were not available from some renal networks.

There was no relationship between centre size and the likelihood of late presenting patients commencing PD (figure 8.9). For any number of patients in the cohort (x-axis), one can identify whether the percentage of patients referred within $<90$ days (y-axis) falls within plus or minus 2 standard deviations (SDs) from the national mean (solid lines, 95\% limits) or 3 SDs (dotted lines, 99.9\% limits). With 43 centres included in the analysis, it would be expected by chance that two centres would fall outside the $95 \%$ ( 1 in 20) confidence limits. The results have to be cautiously interpreted due to the extent and variation in missing data, small numbers of patients in some centres and non-adjustment for any patient related factors.

Figure 8.10 shows that across centres there was large variation in the proportion of PD patients who were late presenters, with many centres not starting any patients presenting at less than 90 days on PD. In two centres, more than $20 \%$ of patients started on PD were late presenters (however one of these centres, Tyrone, only had 3 patients starting PD in 2011).

Figure 8.11 shows first access for centres reporting PD patients in the audit. There was a wide variation between centres in the use of PD as first access. A similar variation is noted in the use of an arteriovenous (AV) fistula as first access. Derby had the highest use of both PD catheter and primary AV fistulas resulting in a less than $20 \%$ use of central lines for the first dialysis. Clearly an understanding of the wide variation between centres 


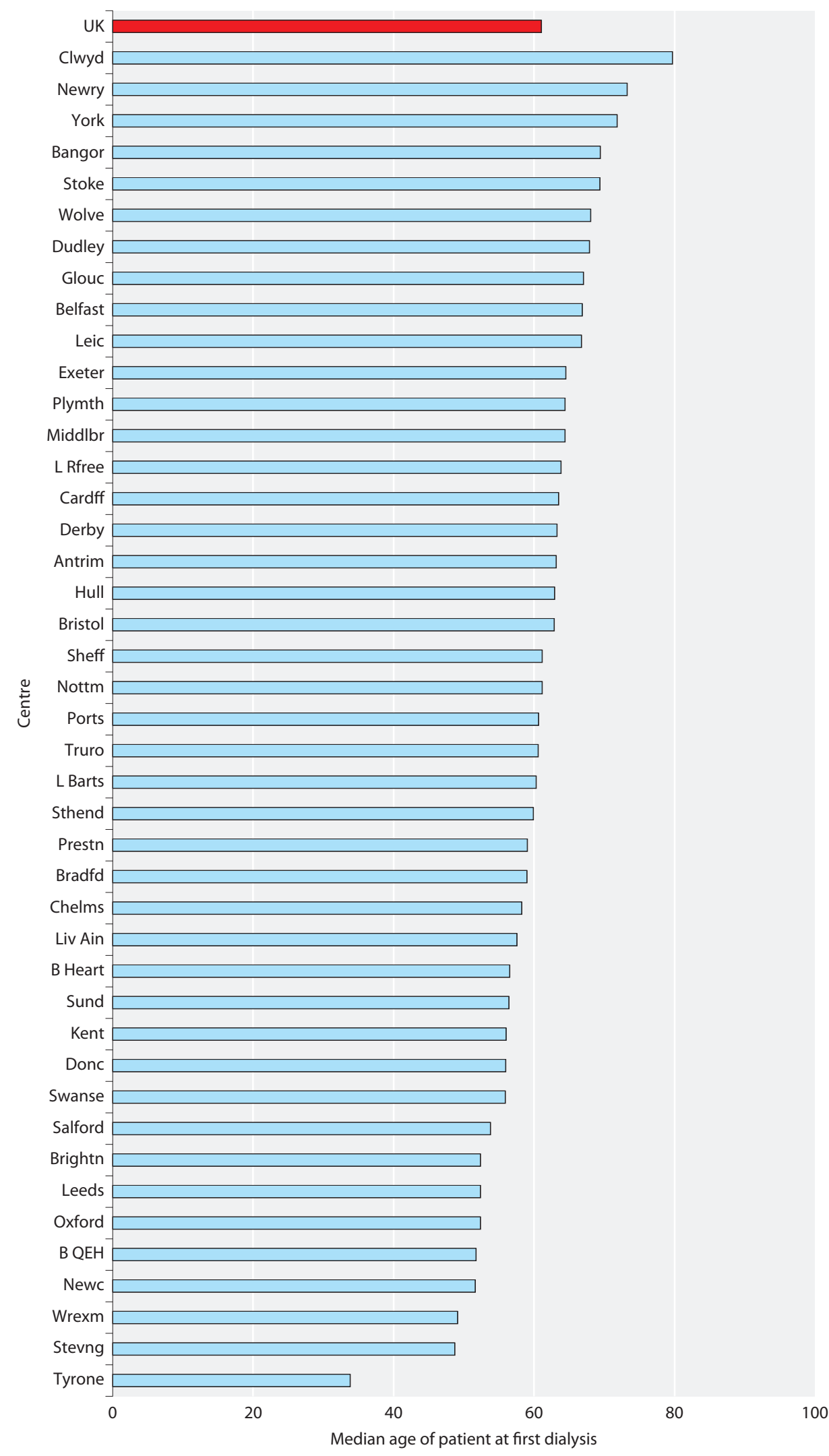

Fig. 8.2. Median age of PD patients at first dialysis by renal centre 


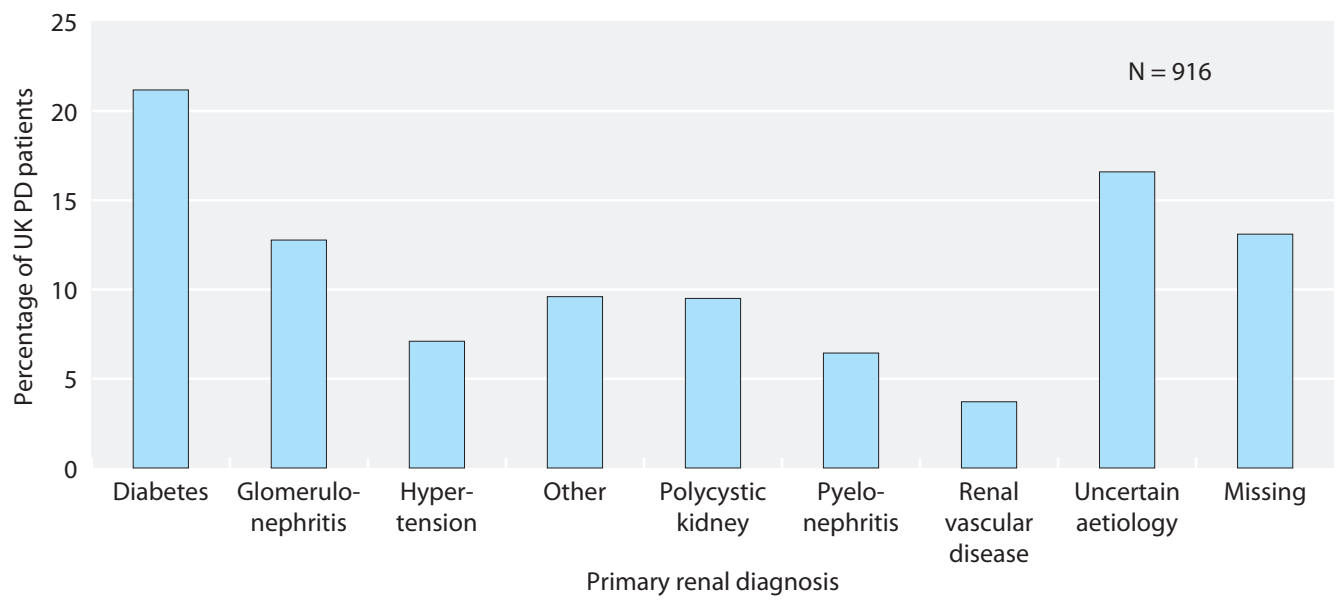

Fig. 8.3. Contributory proportions of primary renal disease in UK PD patients

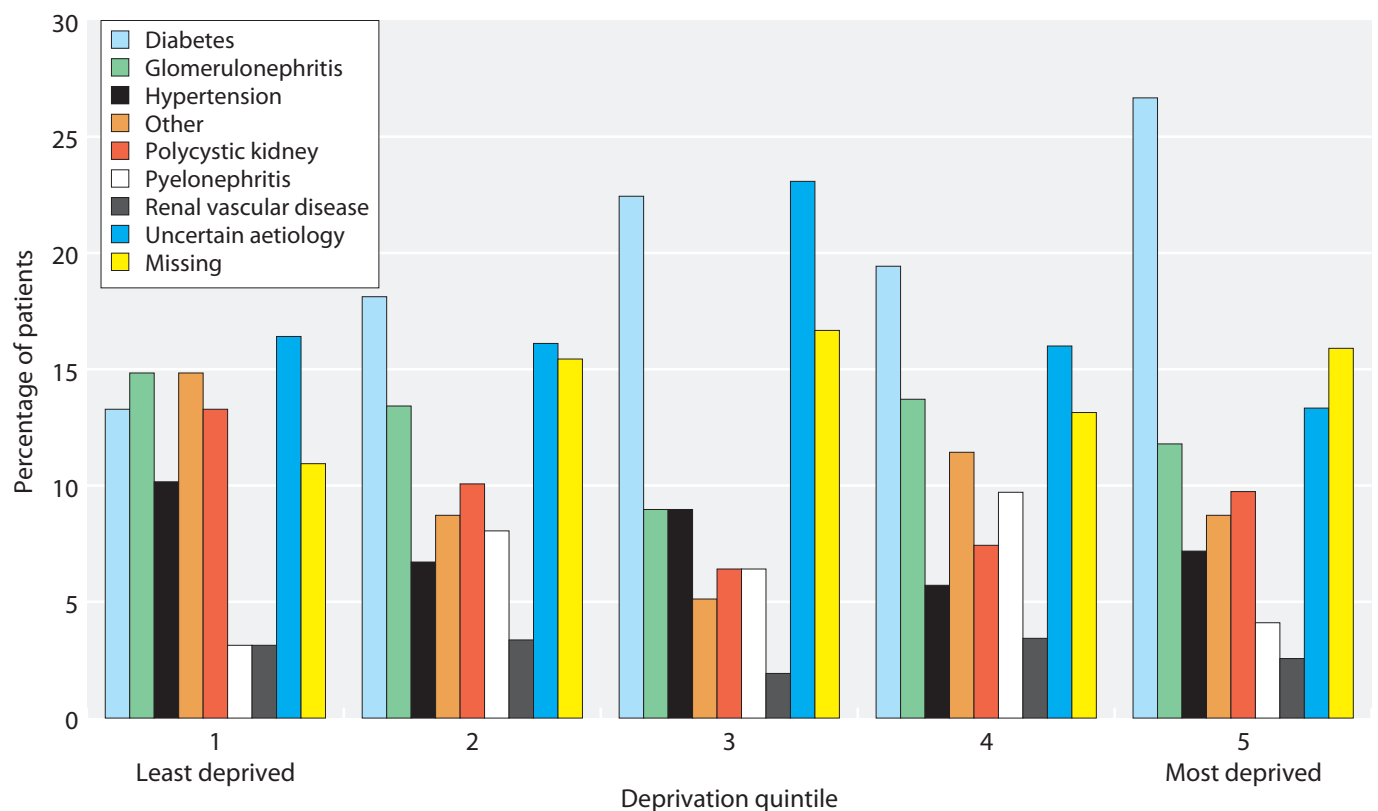

Fig. 8.4. Percentage of patients with each PRD, by deprivation quintile

Based on 803 PD patients with a valid postcode in England (128, 149, 156, 175, 195 patients in the 1-5 quintiles)

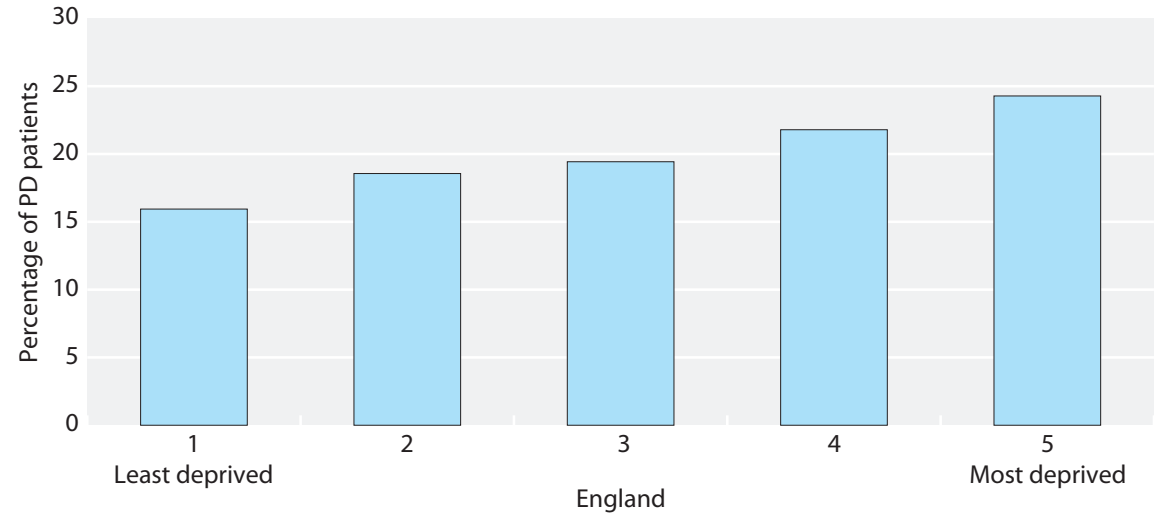

Fig. 8.5. Deprivation quintile profile for $\mathrm{PD}$ patients resident in England Based on 803 PD patients (excludes 113 without valid English postcode) 


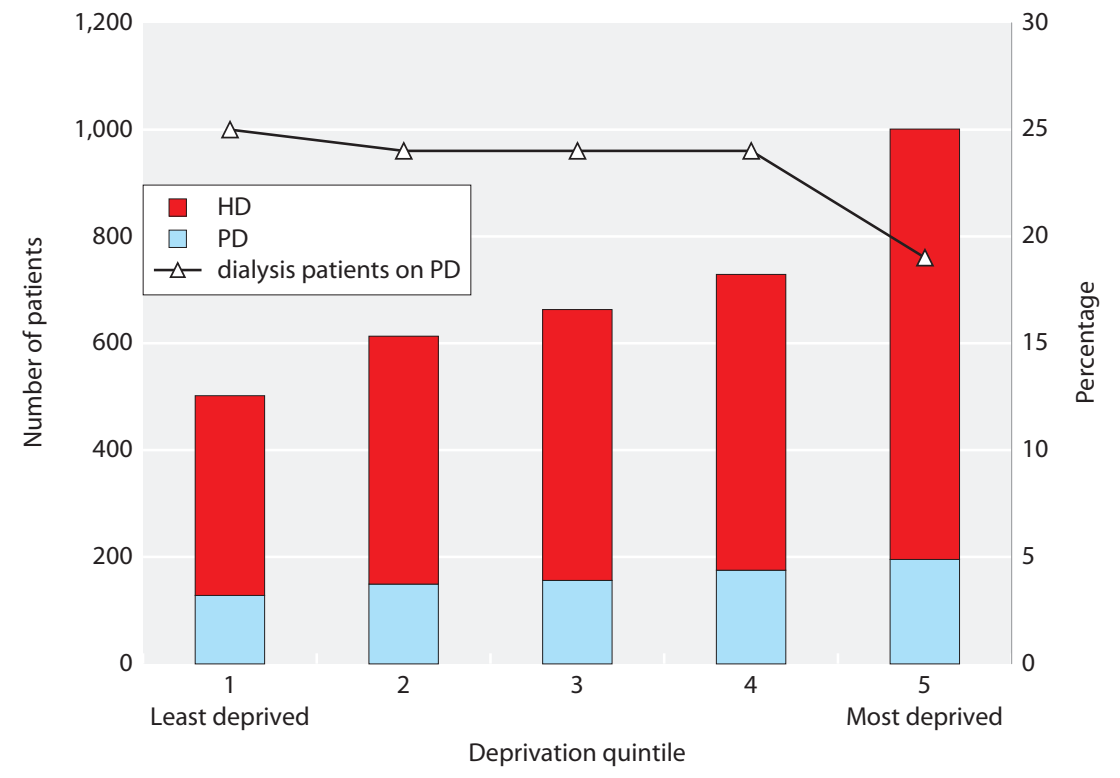

Fig. 8.6. Number and percentage of dialysis patients on $\mathrm{PD}$, by deprivation quintile

Based on 3,508 dialysis patients with valid postcode in England (591 excluded). The bars for $\mathrm{HD}$ and PD represent numbers of patients and relate to the left hand axis. The line graph relates to the right hand axis. is interesting and could lead to potential improvements. There may be reporting differences which need to be explored.

Approximately 50\% of all non-tunnelled lines and 33\% of tunnelled lines were in late presenting patients. The majority of patients who started dialysis with an AV fistula had been known to a physician for over a year - very few late presenting patients were able to start dialysis with either an AV fistula (2\%) or AV graft (6\%) (figure 8.12). The pattern for PD catheter use is similar to that of AV fistulas - where the majority are in patients who have been known to centres for more than 1 year. This suggests that the PD catheter insertion pathway was insufficiently responsive at many centres and that $\mathrm{PD}$ catheters were being under used for late presenters.

From the available data, $75 \%$ of UK renal centre patients who were initiated on PD catheters were documented as PD functioning at three months. Due to small numbers of catheters being inserted at individual centres it is not possible to perform a statistical comparison of three month catheter survival between centres. There was an inverse relationship between lateness of presentation and 3 month retention on PD. Thus $90 \%$ of patients starting therapy less than 90 days from first

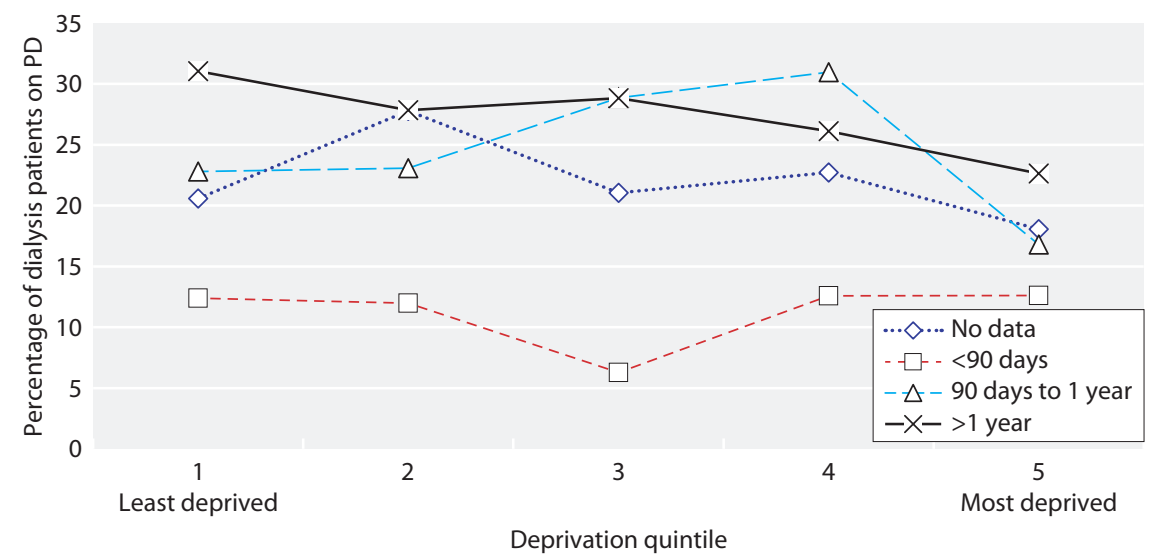

UK Multisite Peritoneal Dialysis Access Catheter Audit
Fig. 8.7. Percentage of dialysis patients on $\mathrm{PD}$ by quintile and length of time between first seeing a renal physician and starting dialysis 


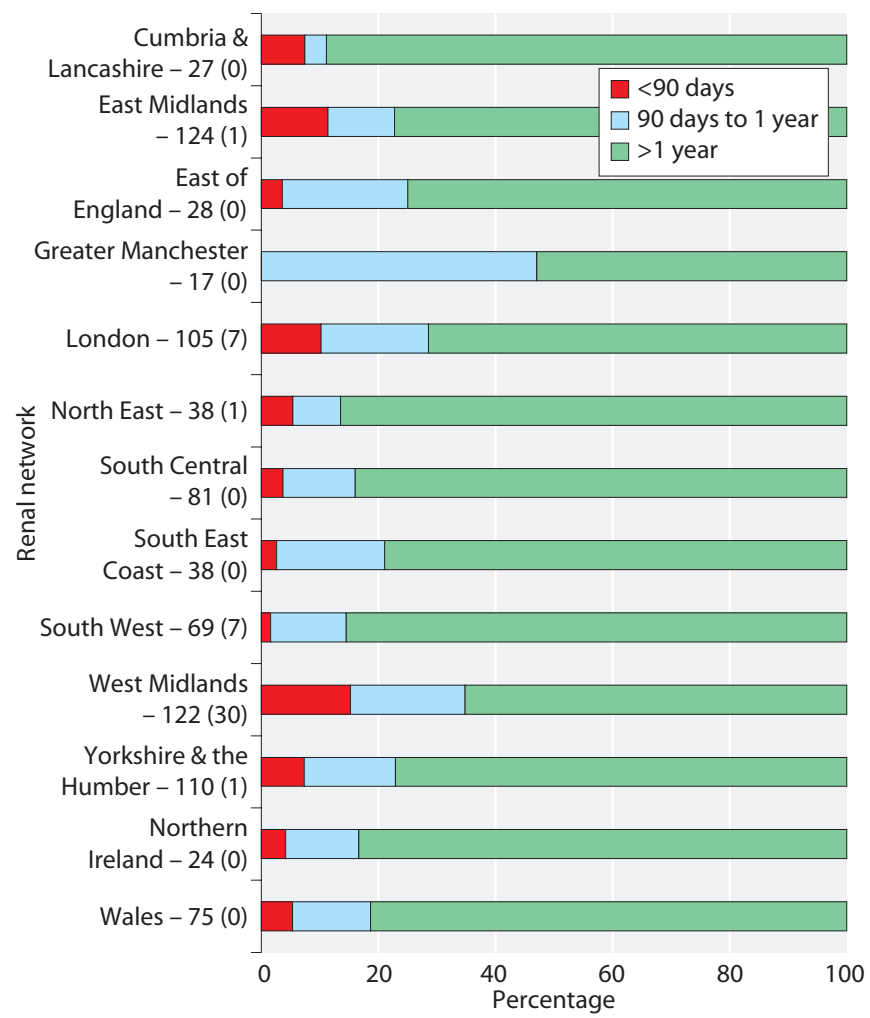

physician contact remained on PD at 3 months; for patients starting PD between 90 days and 1 year following first physician contact, PD utilisation at 3 months was $86 \%$; and for those known to a physician for more than 1 year the 3 month utilisation rate was $75 \%$ (figure 8.13). For patients in whom referral time was not recorded, only $68 \%$ were reported as having a $\mathrm{PD}$

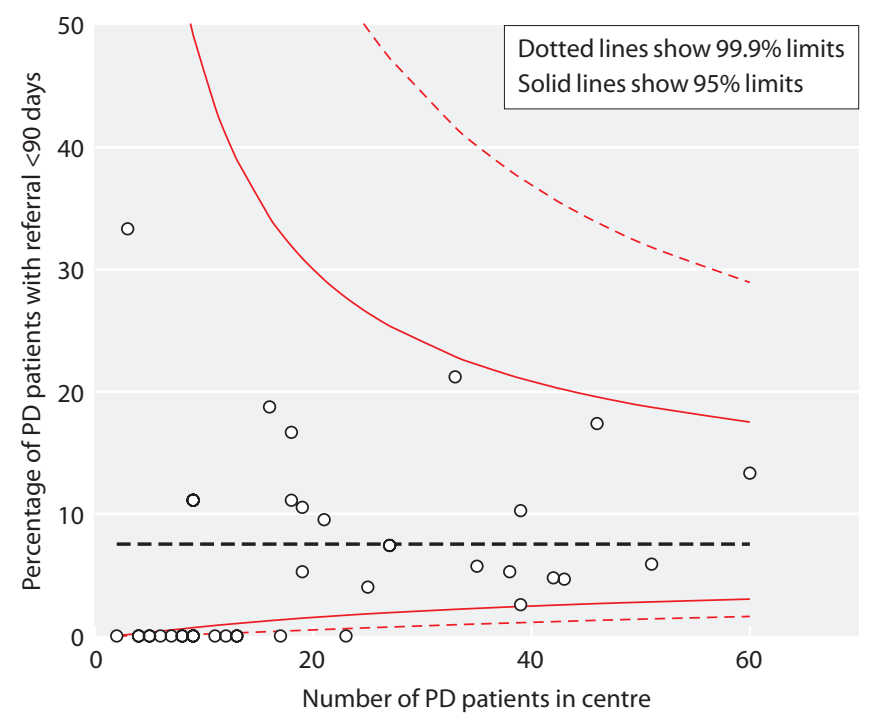

Fig. 8.8. Referral time from first seeing a renal physician to first dialysis for patients starting on PD, by renal network

Based on 867 patients who had PD catheter as first access (number of patients in each network listed on y-axis, includes patients with missing data (number in brackets)). Cheshire \& Merseyside excluded because none of their 9 patients had referral time data returned.

catheter as their access at three months. Thus, there was a greater tendency for patients who started PD 'acutely' to be on PD at 3 months when compared to patients known to the team for longer.

The relationship between PD catheter usage for latepresenters and other access modalities is shown in figure 8.14. The variation in centre practice was wide.

Fig. 8.9. Funnel plot relating number of patients per centre and the percentage of $\mathrm{PD}$ patients with referral $<90$ days 


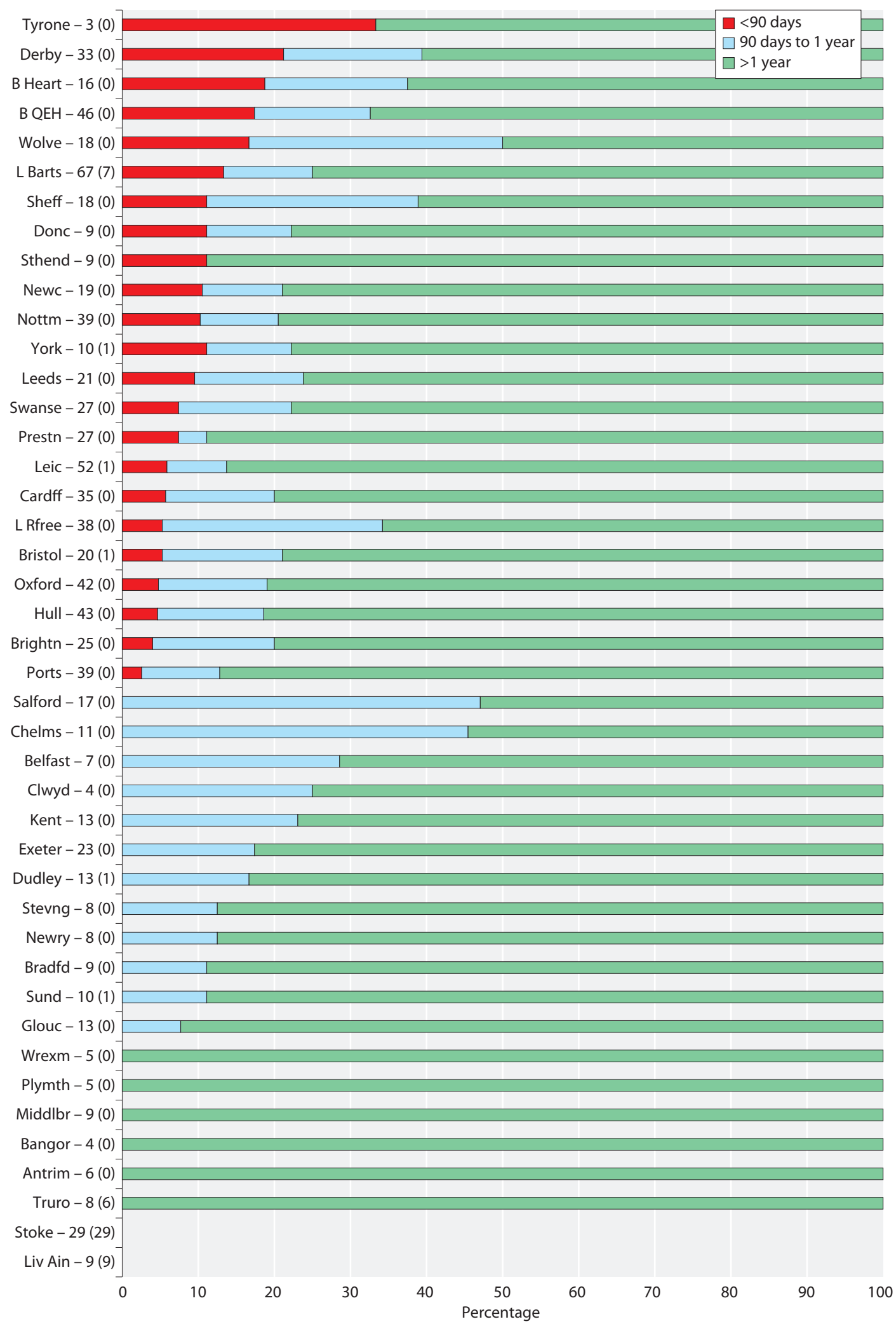

Fig. 8.10. Percentage of PD patients in each referral time category by centre

Based on 867 patients who had PD catheter as first access (number of patients at each centre listed on y-axis), includes patients with missing data (number in brackets) 


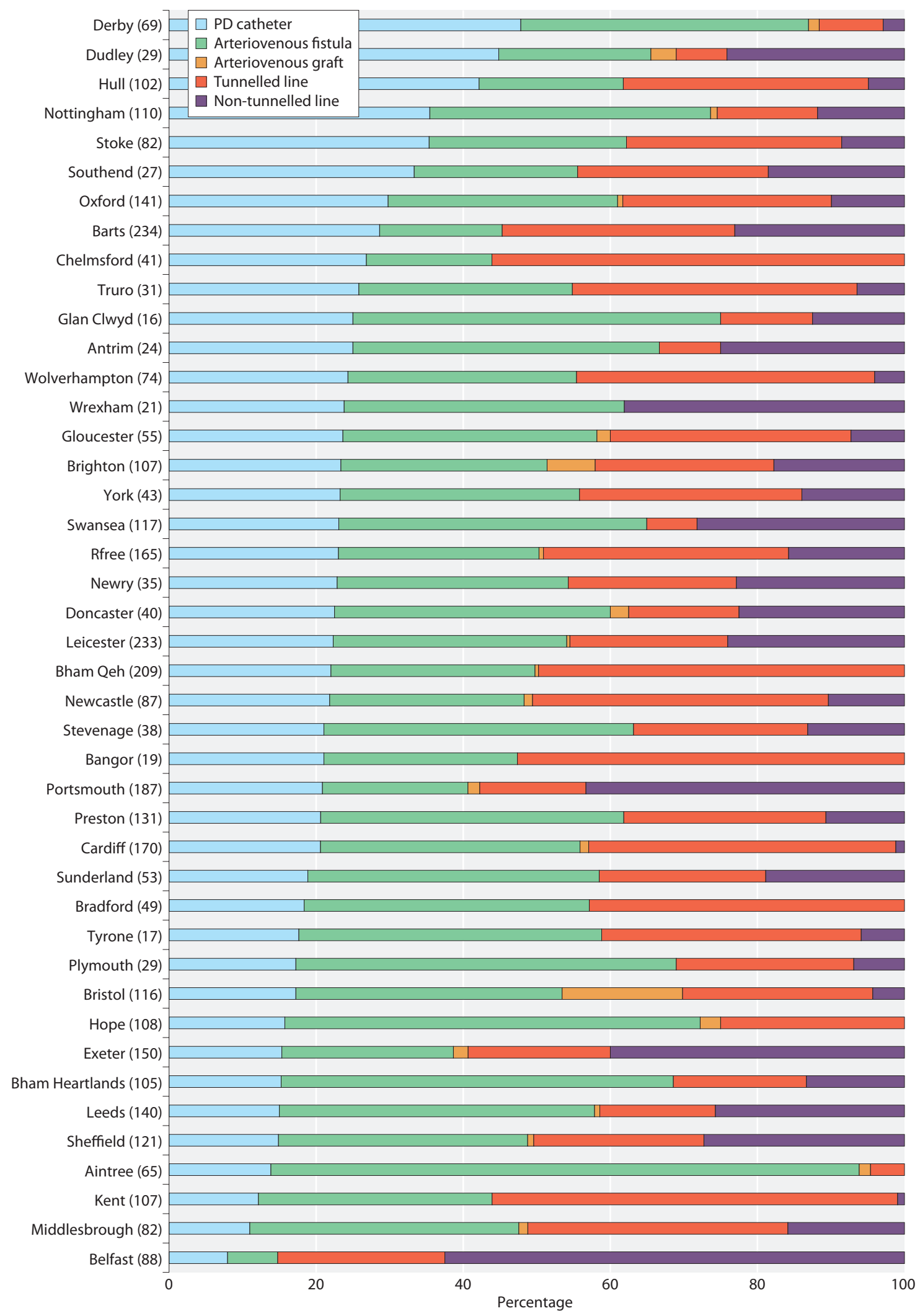

Fig. 8.11. Access at first dialysis for centres reporting PD patients, by renal centre

Based on 3,867 dialysis patients from centres that reported PD patients. Number of patients at each centre in brackets. 


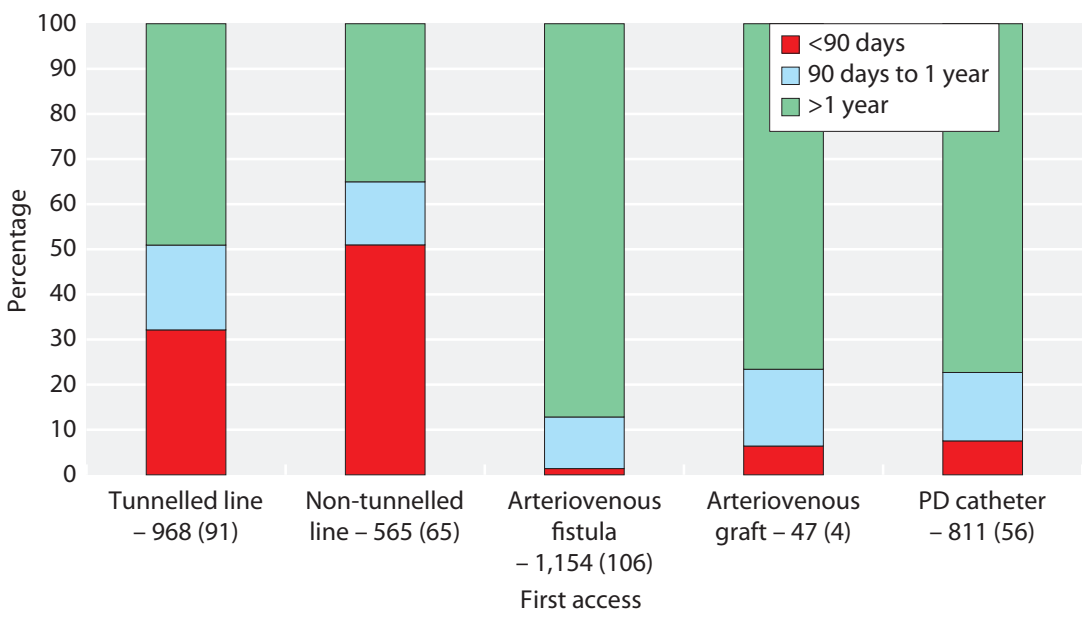

Fig. 8.12. Referral time from first being seen by renal physician to starting dialysis by type of first access Based on 3,545 patients from centres that reported PD patients, who had data on both referral time and type of first access. Total number of patients contributing data to the chart by access type included in $\mathrm{x}$-axis labels (number with missing data in brackets).
For example, Derby used PD catheters in $58 \%$ of late presenting patients with tunnelled lines in $25 \%$, when compared with Chelmsford where all late presenters commenced dialysis via tunnelled catheters. Belfast had 26 and Exeter 43 patients classed as late presenters with the majority of patients commencing dialysis via a non-tunnelled line. Again, differences in reporting practice of first access may be important.

There was wide variation in the practice of surgical assessment for PD catheter insertion (figure 8.15). Surgical assessments varied from $0 \%$ in 5 of the centres to $100 \%$ in Antrim, Birmingham QEH and Bangor. This likely reflects the differing surgical services in renal centres. Throughout the UK, approximately half of all patients where data were returned were assessed by a surgeon before PD catheter insertion. Many centres did not report this data.

Late presenters initiated on PD were less likely to undergo surgical referral for PD catheter insertion. For late presenters to be established on $\mathrm{PD}$, a responsive pathway is essential, and to this end medical PD catheter insertion allows the nephrologist to have control over the process. Patients who had been known to the services longer were more likely to be referred to a surgeon, although there was considerable missing data (figure 8.16).

Information regarding the use of insertion technique stratified by advanced surgical assessment was compromised by missing data in 375 patients. An association was noted between surgical assessment and open surgical catheter placement, which is unsurprising.
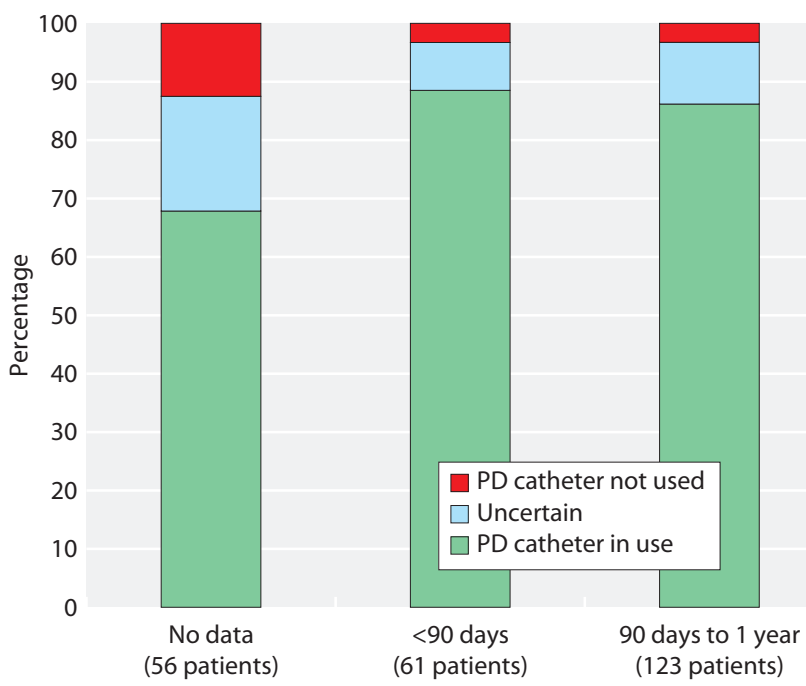

Referral time
Fig. 8.13. Access at 3 months after starting peritoneal dialysis, by referral time Based on 867 patients who had PD as their first access 


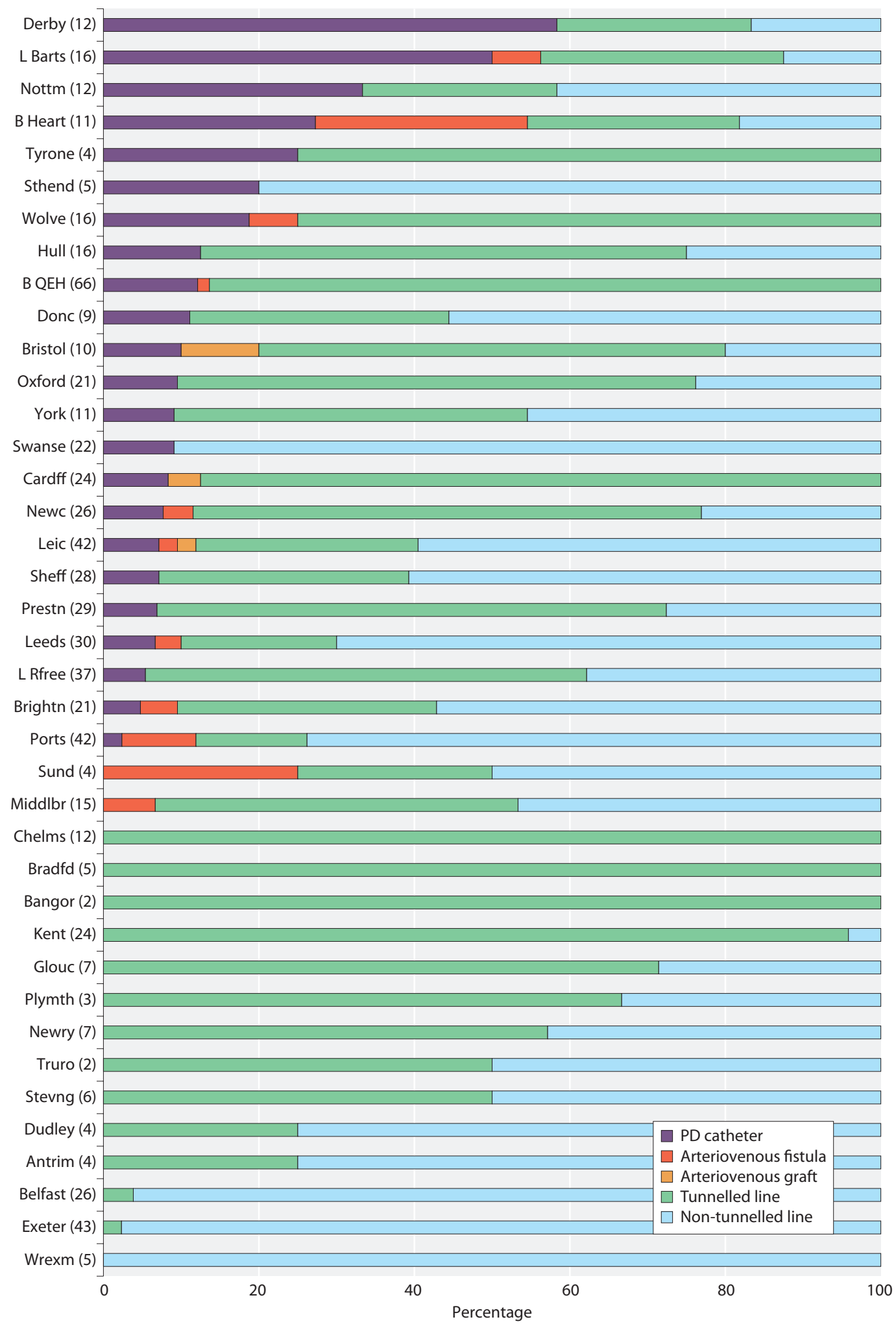

Fig. 8.14. Type of first access for late presenting patients (referral time of $<90$ days) by centre Based on 750 patients who had less than 90 days between when they were first seen by a physician and starting dialysis (individual centre totals in brackets). 4 centres do not appear on the chart. Aintree and Stoke returned no data for referral time. Salford and Glan Clwyd had no patients who had a referral time of less than 90 days. 


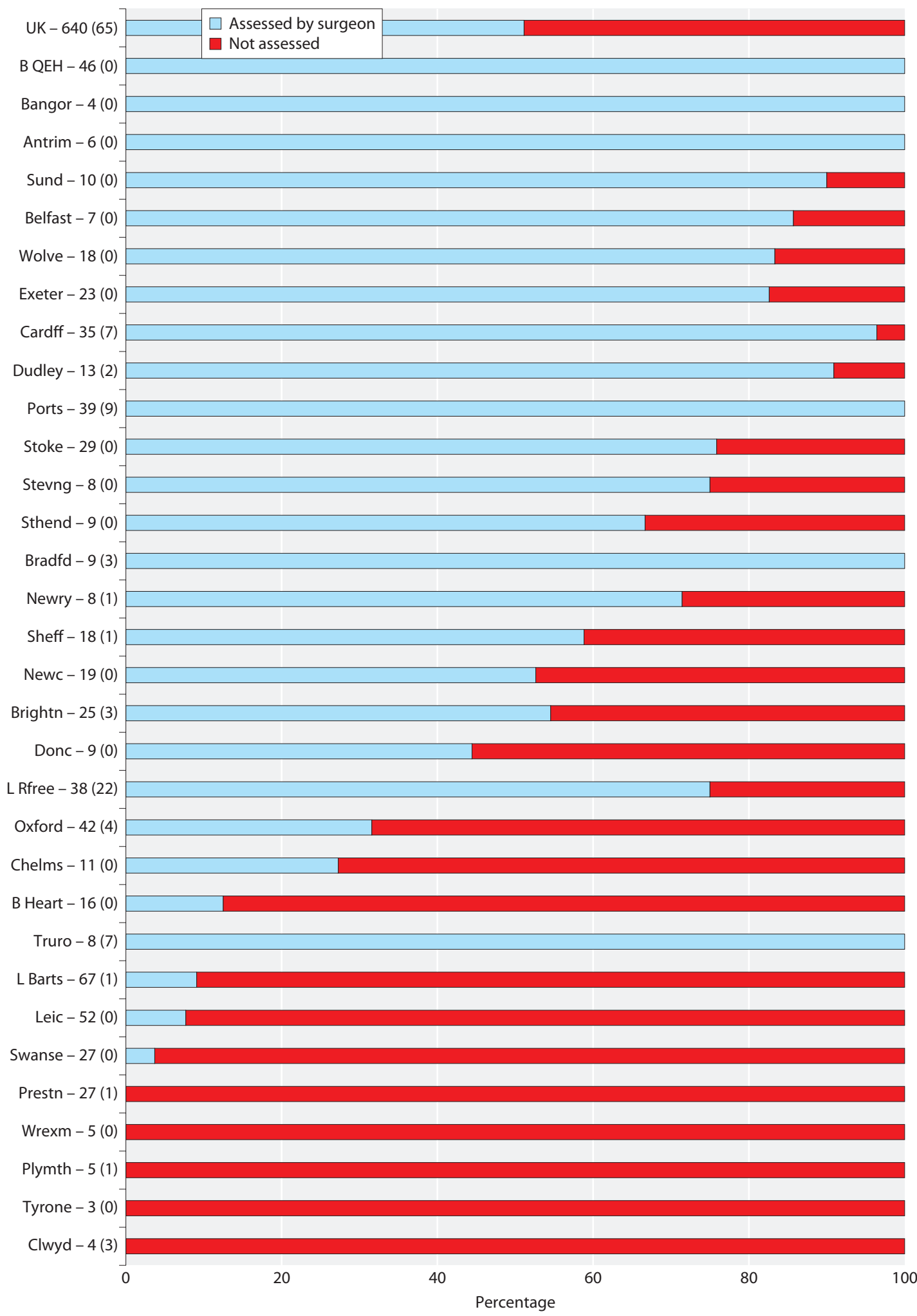

Fig. 8.15. Assessed by surgeon, by renal network for patients with first access as PD catheter

Based on 640 patients who had PD as their first modality. Total number of patients listed after centre name (number of patients with missing data in brackets). The following centres are not displayed as they did not return any data: York, Nottingham, Middlesbrough, Leeds, Kent \& Canterbury, Hull, Manchester, Gloucester, Derby, Bristol and Liverpool Aintree. A total of 227 patients were excluded from these centres. 


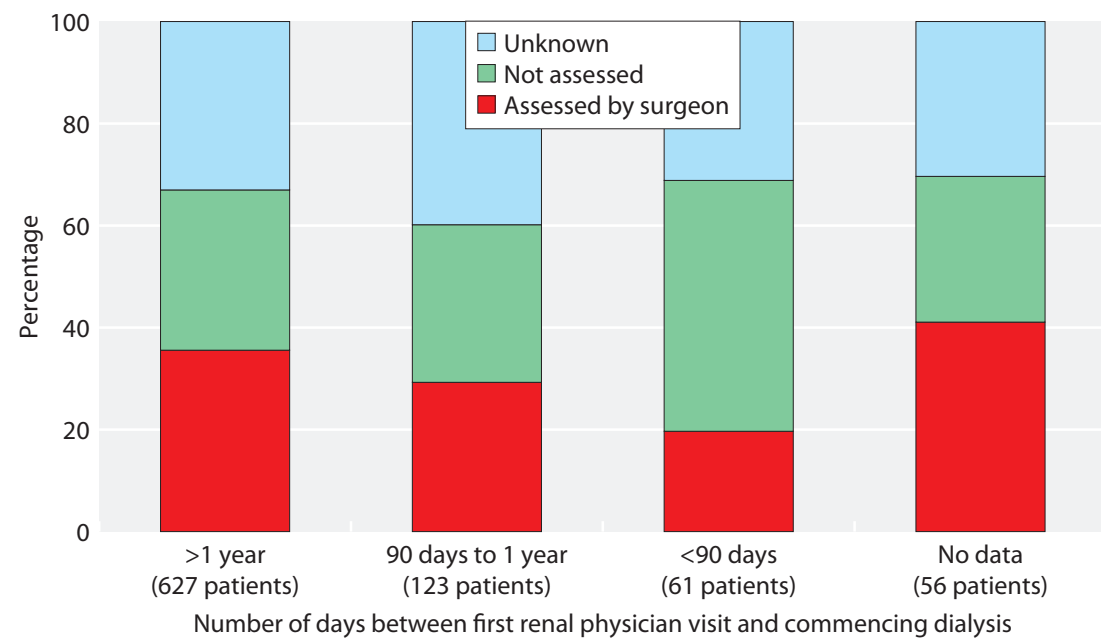

Fig. 8.16. Assessed by surgeon, by referral period for patients with PD as first access
The practice of surgical assessment three months in advance of PD catheter insertion varied between centres with 294 patients in the UK who received a PD catheter being seen by a surgeon and 281 reported as not being seen (figure 8.17).

PD catheter insertion technique varied in accordance with the time to PD initiation from first physician contact (figure 8.18). There was greater representation of percutaneous insertion in so-called 'late presenters'. Of late presenting patients, $40 \%$ had the PD catheter inserted by the percutaneous method, this figure fell to $30 \%$ in patients presenting between 90 days and 1 year prior to dialysis start and $28 \%$ in patients known to

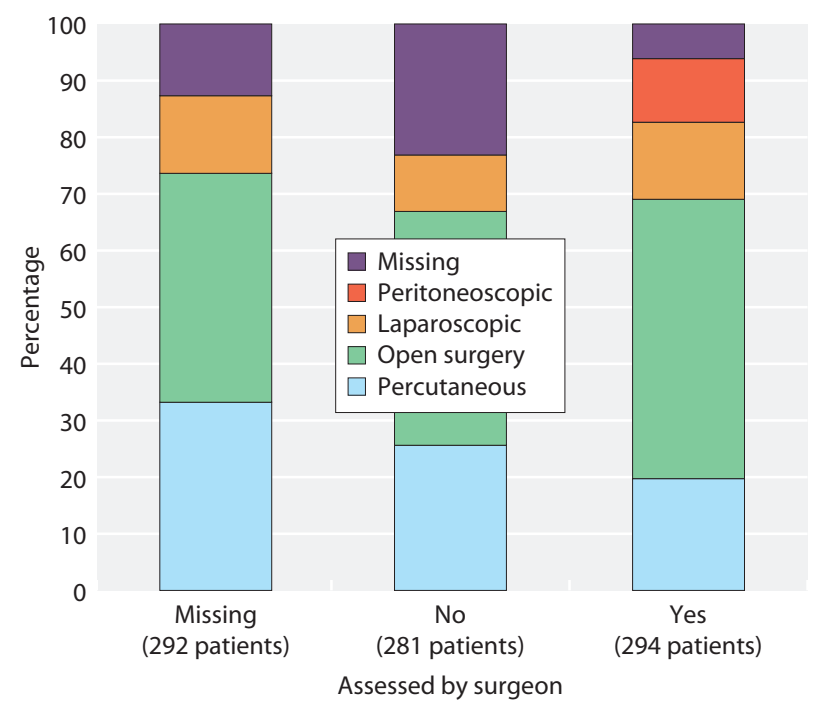

Fig. 8.17. PD catheter insertion technique by surgical assessment the service for more than 1 year.

\section{$P D$ catheter outcomes}

The number of patients with sufficient data to be included in this analysis was low (121 patients), however there appeared to be more catheter flow problems with percutaneous catheters (20 out of 38) compared with 23 out of 62 catheters placed by the open surgical technique (figure 8.19). Outcomes were reported in only 17 laparoscopic cases and 4 peritoneoscopic cases and therefore no conclusions can be drawn for these

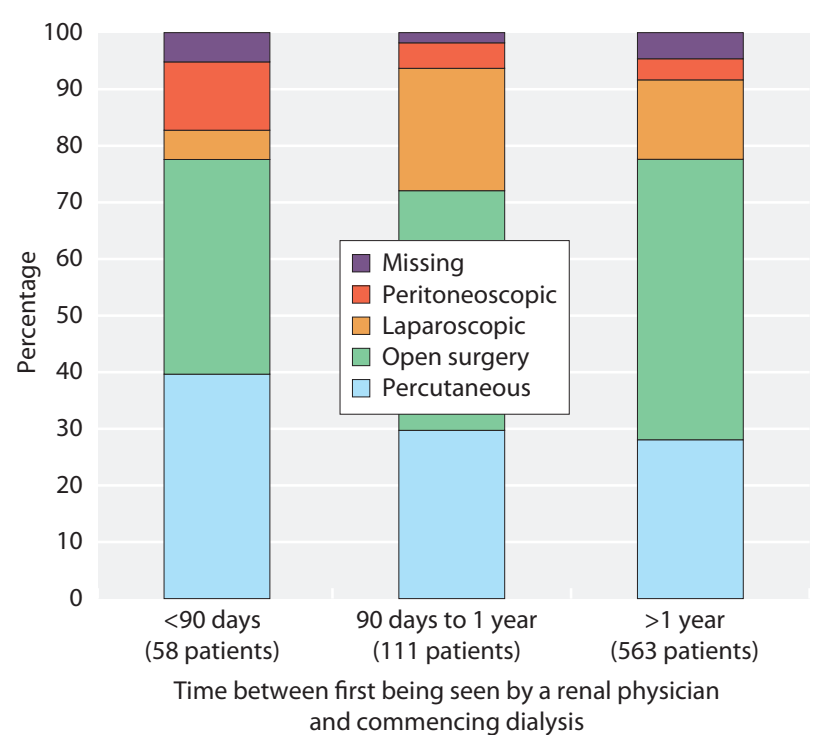

Fig. 8.18. $P D$ catheter insertion technique by referral time Based on 732 patients who had PD catheter as their first access and a valid referral time. Six centres were excluded, two due to returning no data about referral time and four due to returning no data about insertion technique. 


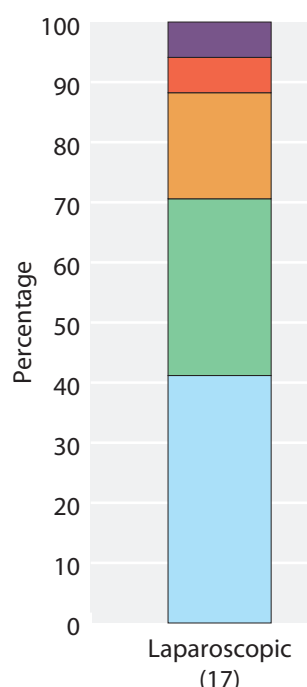

(17)

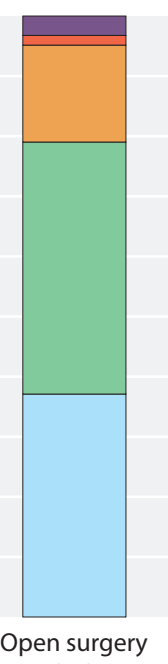

(62)

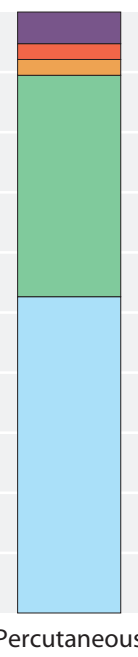

(38)

Catheter insertion technique

\section{techniques.}

Figure 8.20 describes catheter complications according to whether the patient was described as having diabetes (type $1 \& 2$ ) at the time of catheter insertion (as distinct from diabetes as the primary renal disease). Of the 916 patients, 224 were recorded as diabetic, 483 as not diabetic and 209 had missing data. Patients with diabetes may be anticipated to have higher rates of infection and thus catheter failure at 3 and 12 months. Although the numbers of patients with diabetes included in this analysis were small (112 patients had complete data) there was no excess of complications in these patients compared to those without diabetes. There

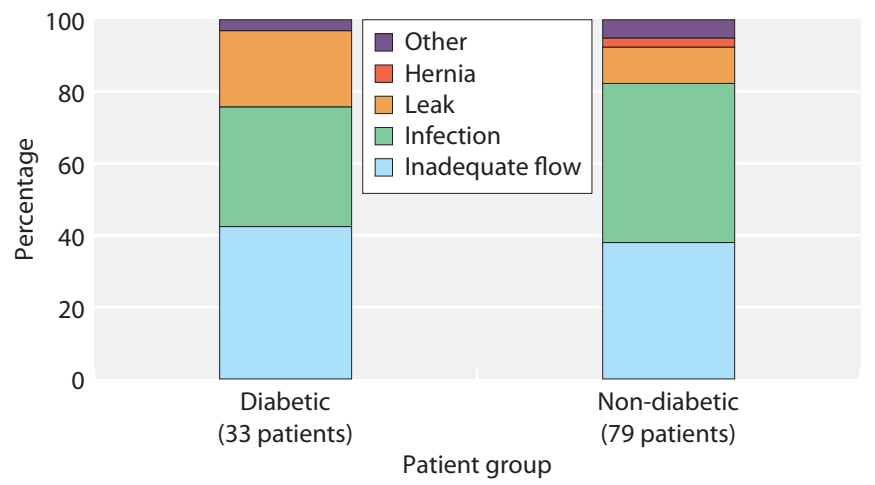

Fig. 8.20. Reason for catheter failure, diabetic vs. non-diabetic Seven centres (206 patients) excluded due to poor data completeness $(<50 \%$ of patient records returned with data on diabetes status at time of catheter insertion). No catheter failure recorded for 580 patients. No failure reason recorded for 16 patients who had a failure date recorded. Two patients with no data returned about diabetes status have a failure reason recorded as inadequate flow-inflow and outflow

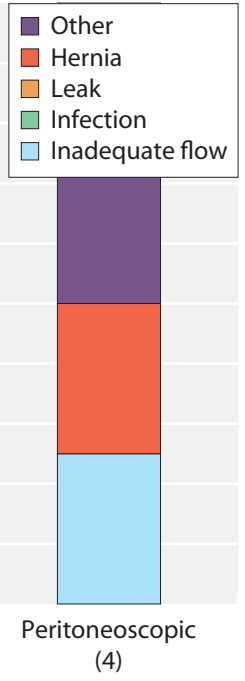

Fig. 8.19. Catheter failure reason by insertion technique

Based on 121 patients. Number of failures by insertion technique in brackets. Inadequate flow covers inflow and outflow.

was no association between diabetes and the likelihood of early peritonitis (less than 2 weeks) however a number of patients (283) were excluded due to poor data completeness (figure 8.21).

The Renal Association Guidelines suggest exit site infection within 2 weeks of catheter insertion should occur in less than $5 \%$ of patients and also that peritonitis within 2 weeks of catheter insertion should be similarly rare. There was a significant difference $(p=0.016)$ in the percentage of catheters recorded as experiencing an

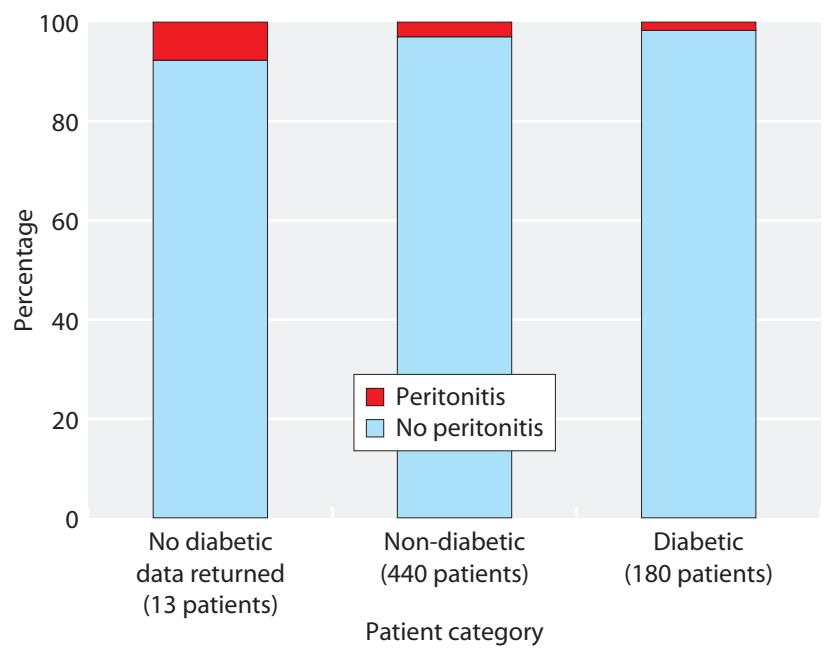

Fig. 8.21. Percentage of patients who experienced a peritonitis episode within 2 weeks of catheter insertion

Ten centres had all their patients (270) excluded due to poor data completeness $(<50 \%$ completeness for either the diabetes or peritonitis fields). 13 additional patients excluded from chart as no data returned regarding peritonitis. 


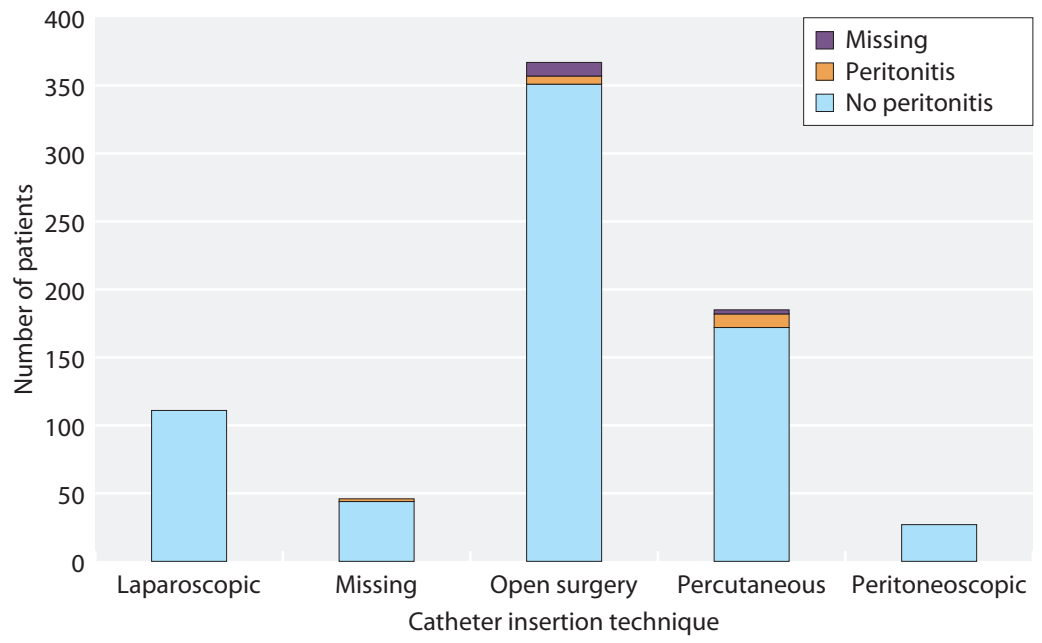

Fig. 8.22. Number of patients who experienced a peritonitis episode within 2 weeks of catheter insertion, by catheter insertion technique 180 patients excluded due to poor data completeness at their centre $(<50 \%$ with peritonitis field completed) early peritonitis episode (at less than 2 weeks post catheter insertion) for each insertion technique. The highest percentage of early peritonitis episodes for a technique was $5.4 \%$ for percutaneous catheter insertions which was at the level of the audit standard (5\%) (figure 8.22). The majority of patients underwent open surgical PD catheter insertion.

Catheter failure by 3 months was most common amongst patients with percutaneous catheters, 31 failures of 202 inserted (15.3\%). The other insertion techniques reported on were open surgery, 33 failures of 380 inserted (8.7\%); laparoscopic, 8 failures of 111 inserted (7.2\%); and peritoneoscopic, 2 failures of 33 inserted (6.1\%) (figure 8.23). A log-rank test found some evidence $(\mathrm{p}=0.039)$ of a difference between the catheter survival distributions for the four insertion techniques.

\section{Conclusions}

This is the first multisite PD catheter audit in the UK. It highlights a number of important points including:

- Peritoneal dialysis is less likely to be used compared with haemodialysis for those from geographical areas with higher deprivation scores.

- There was wide variation between centres of PD catheter use for late presenting patients (known to centres for less than 90 days).

- The percutaneous PD catheter insertion technique was more commonly used than the open surgical technique for late presenting patients.

- Overall, patients are more likely to get a PD catheter if they had been known to the service for more than 1 year.

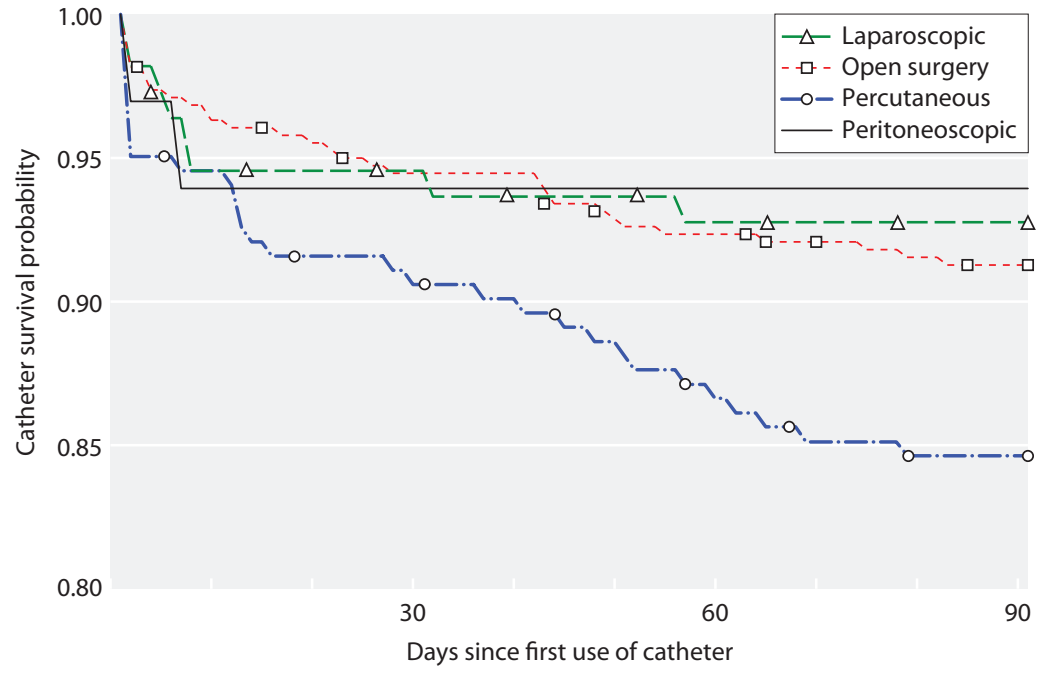

Fig. 8.23. Catheter survival by insertion technique 
- The percutaneous insertion technique was associated with a higher early (less than 2 week) peritonitis rate and more catheter flow problems.

- Patients with diabetes did not have an increased complication rate following PD catheter insertion.

\section{Recommendations}

1. The prospective collection of information relating to dialysis is central to understanding quality of care in this area and should be supported to continue and develop.

2. Centres need clear systems to report PD catheter access to the UK Renal Registry. At present too few of the Electronic Patient Record systems in use in renal centres lend themselves to the easy capture of this data. Clinical Directors need to discuss the support of these and related tools to allow national audit more seamlessly than currently happens.

3. Attention should be given to the pathway of catheter placement. The evidence from this audit that patients are more likely to get onto PD if known more than 1 year to the service indicates that the processes around PD initiation are too slow at most centres and represent a missed opportunity. However some centres have demonstrated that with the appropriate structures, this process can be speeded up and late presenting dialysis patients can be started on PD. Many of the patients who do not get PD catheters end up with tunnelled or non-tunnelled haemodialysis lines with which there is evidence of poorer outcomes [10].

4. Centres should employ quality assurance measures to ensure that the success of PD catheter placement is monitored locally [11].

\section{Acknowledgements}

Thanks are expressed to the Healthcare Quality Improvement Partnership who have funded this audit in conjunction with the UK Renal Registry; members of the PD Access Audit Group including their patient partners; and Richard Fluck as vascular access group lead.

Conflicts of interest: none

\section{References}

1 Gilg, J, Castledine C, Fogarty D. Chapter 1 UK RRT incidence in 2010: national and centre-specific analyses. Nephron Clin Pract, 2012. 120 Suppl 1: p c1-27

-2 Figueiredo A, et al. Clinical practice guidelines for peritoneal access. Perit Dial Int, 2010. 30(4): p 424-9

3 Mactier R, et al. Summary of the 5th edition of the Renal Association Clinical Practice Guidelines (2009-2012). Nephron Clin Pract, 2011. 118 Suppl 1: p c27-70

4 Briggs V, et al. Getting more out of clinical practice guidelines. Perit Dial Int, 2011. 31(6): p 631-5

5 Woodrow, G and S Davies, Renal Association Clinical Practice Guideline on peritoneal dialysis. Nephron Clin Pract, 2011. 118 Suppl 1: p c287-310

-6 Briggs V, Wilkie M. Chapter 14 Comparative audit of peritoneal dialysis catheter placement in England, Northern Ireland and Wales in 2011: a summary of progress to July 2012. Nephron Clin Pract, 2012. 120 Suppl 1: p c261-3

7 Report Of The UK Renal Association Working Party On Peritoneal Access. 2008

8 Fluck RPD, Steenkamp R. Vascular access report 2012. 2012, Renal Registry and NHS Kidney Care

$\checkmark$ Caskey FJ, et al. Social deprivation and survival on renal replacement therapy in England and Wales. Kidney Int, 2006. 70(12): p 2134-40

10 Perl J, et al. Hemodialysis vascular access modifies the association between dialysis modality and survival. J Am Soc Nephrol, 2011. 22(6): p 1113-21

11 Goh BL, Ganeshadeva Yudisthra M, Lim TO. Establishing learning curve for Tenckhoff catheter insertion by interventional nephrologist using CUSUM analysis: how many procedures and in which situation? Semin Dial, 2009. 22(2): p 199-203 